\title{
INVESTIGACIÓN GEOTÉCNICA PARA LA RED DEL METRO DE LIMA
}

\section{GEOTECHNICAL RESEARCH FOR LIMA METRO NETWORK}

\author{
Jorge D. Coll Calderón*
}

RECEPCIÓN: SETIEMBRE DE 2017

ACEPTACIÓN: OCTUBRE DE 2017

\begin{abstract}
RESUMEN
La investigación geotécnica ejecutada para los estudios de las líneas subterráneas 2, 3 y 4 del Metro de Lima es insuficiente como para poder obtener una buena predictibilidad del presupuesto de obra. Además, se ejecutaron ensayos que no permiten definir las propiedades mecánicas de las gravas de Lima-aquellas que contienen canto rodado- de una manera racional. Por tanto, se recomienda un plan de investigación geotécnica especial que consiste en la ejecución de ensayos adecuados para poder determinar las propiedades mecánicas de las gravas, sobre todo en materia de cohesión, que es una variable importante en el caso de excavaciones. En esta investigación, se trataría de buscar correlaciones entre las propiedades mecánicas y ciertas propiedades índice a escogerse, las cuales son mucho más económicas. De esta manera, la investigación geotécnica masiva y lineal a lo largo de cada línea de Metro se abocaría a la obtención de abundante data de las propiedades índice para obtener propiedades mecánicas de una manera indirecta con la ayuda de las correlaciones obtenidas, previamente, en la investigación geotécnica especial.
\end{abstract}

Palabras clave: predictibilidad del presupuesto de obra, canto rodado, propiedades índice, nivel freático, resistencia al corte pico, resistencia al corte residual.

\begin{abstract}
The geotechnical investigation executed for the studies of the underground lines 2, 3 and 4 of the Lima Metro, are insufficient in quantity, so as to obtain a good predictability of the work budget; and in addition, tests were carried out that do not allow to define the mechanical properties of the Lima gravel (which contains boulder) in a rational way. Therefore, a "special geotechnical research" plan is suggested, which consists in the execution of special and expensive tests, but which are adeaquate to be able to determine the mechanical properties of the Lima gravels, and above all, of the cohesion, which is an important variable in the case of excavations works. The goal for this "special geotechnical investigation", it is looking for correlations of the mechanical properties with certain Index Properties to be chosen, properties that are much more economical to obtain. In this way, the massive and linear geotechnical research along each Metro line, would aim to obtain abundant data from the Index Properties, in order to obtain the mechanical properties, in an indirect way, and with the help of the previously obtained correlations in the "Special Geotechnical Investigation".
\end{abstract}

Keywords: predictability of the work budget, boulder, index properties, water table, peak shear strength, residual shear strength. 


\section{Contexto geológico de Lima Metropolitana}

La ciudad de Lima Metropolitana se asienta, principalmente, en el cono aluvial del río Rímac, y se extiende hasta tocar el valle del río Lurín por el sur, y el valle del río Chillón por el norte. La mayor parte de la futura Red del Metro de Lima (RML) ocuparía la zona central del cono aluvial del río Rímac (ver fig. 1), la misma que está compuesta por una grava muy compacta sin presencia del nivel freático $(\mathrm{NF})$ en la profundidad de interés $(<50 \mathrm{~m})$, y que es homogénea en su granulometría, pero no necesariamente en su capacidad mecánica. Los bordes del cono aluvial (Callao, Chorrillos) están compuestos por un material más heterogéneo -además de arena SM y arcilla CL- y en estado menos compacto. Estos son sectores donde, precisamente, ocurrieron los mayores daños a consecuencia de los grandes terremotos del siglo XX. En el Callao, el suelo es tanto menos denso cuánto más cerca está del mar. Además, cuenta tiene el NF cada vez más superficial [1] . Cabe mencionar que, de acuerdo con la tomografía de las velocidades de ondas de corte a lo largo de la Av. Colonial (L2 de este a oeste), se muestra un cambio brusco en el eje de la Av. Faucett, con un suelo muy denso y sin NF hacia el este (provincia de Lima), y un suelo medianamente denso y con NF hacia el oeste (provincia de Callao). También, cabe mencionar que no se conoce la profundidad del basamento rocoso en el valle de Lima, pero se tiene una estimación cualitativa del perfil estratigráfico hasta este basamento (ver fig. 2).

\section{Cantidad y tipo de investigación geotécnica realizada}

Para conseguir una predictibilidad aceptable del presupuesto de obra, se requiere, previamente, invertir en investigación geotécnica, al menos en el margen del 1\% a 4\% del monto de la obra. Obviamente, tanto menos cuanto el suelo es bueno y homogéneo; y viceversa [2]. Sin embargo, para el estudio a nivel de preinversión de la L2 del Metro, se invirtió apenas el 0.1\%; y para las líneas L3 y L4, se incrementó, apenas, al $0.4 \%$ [3].

Las propiedades mecánicas son las variables más importantes a obtener en la investigación geotécnica, pues permitirán diseñar las estructuras subterráneas (estaciones y túneles) de manera óptima (más económica). Estas propiedades mecánicas se pueden obtener, para las arenas SM y arcillas CL, mediante ensayos estándar de campo y laboratorio, tales como ensayos SPT, ensayos de corte sobre muestras inalteradas, etc. [4]. Estos ensayos no son aplicables a las gravas GP, que es el suelo, ampliamente mayoritario, para la RML. No obstante, se ejecutaron, inútilmente, varios ensayos SPT para el estudio de la L2 [1]; y, para el estudio de la L3 y L4, no se ejecutaron los ensayos especiales que se requerían, por lo menos, de manera suficiente [3].

\section{Propuesta de investigación geotécnica}

El tamaño de una muestra de suelo sometido a un ensayo de resistencia al corte debe ser, al menos, de 5 a 6 veces el tamaño de la partícula más grande que la contiene a fin de que esta no distorsione los resultados [5]. De esta manera, según los análisis granulométricos realizados en la grava GP para los estudios de las líneas de Metro, y debido a la presencia de canto rodado, el tamańo requerido de una muestra de suelo sometido en un ensayo de resistencia al corte debiera ser del orden de $70 \mathrm{~cm}$ a 200 $\mathrm{cm}$, lo que evidentemente implicaría la necesidad de ejecutar ensayos especiales que no están normalizados y que serían muy caros. Por lo tanto, sería necesario ejecutar estos ensayos especiales dentro de un programa de investigación geotécnica especial (IGE), de tal manera que se busquen correlaciones de las propiedades mecánicas con las propiedades índice [6]. En la investigación geotécnica, las propieda- 
des índice son, generalmente, económicas. Así, en la investigación geotécnica masiva (IGM) o lineal a lo largo de cada línea de Metro, se determinaría las propiedades índice para luego usar las correlaciones previamente determinadas en la IGE y, de este modo, obtener indirectamente las propiedades mecánicas que se requieren para los diseńos [6]. Los detalles de la IGM e IGE se describen más adelante.

Desde finales de la década de los 70, se vienen ejecutando ensayos de corte directo in situ en caja grande (ver fig. $3: 60 \mathrm{~cm}$ a $70 \mathrm{~cm}$ ). Estos suelen realizarse de manera esporádica, aunque, últimamente, debido a la implementación de la RML, se vienen ejecutando de manera más intensa. El problema con estos ensayos es que, al tallar las muestras, no se sabe a priori cuál es la granulometría y, sobre todo, el tamaño de la partícula de más grande incrustada en lo que sería el futuro plano de falla. Por lo tanto, no hay control sobre la reproductibilidad de las muestras en los diferentes ensayos. A consecuencia de esto, los resultados de la resistencia al corte obtenidos in situ muestran resultados en un rango muy amplio (ver tabla 1), cuando es de esperar que no ocurra así para un suelo aparentemente homogéneo en cuanto a su clasificación [6].

TABLA 1. RANGo DE VAlORES DE LA RESISTENCIA AL CORTE IN SITU OBTENIDAS PARA LA GRAVA COMPACTA DE LIMA EN BASE A I 5 ENSAYOS

\begin{tabular}{|l|c|c|c|}
\hline & $\tau_{\text {máx }}$ & $\tau_{\text {promedio }}$ & $\tau_{\min }$ \\
\hline Ángulo de fricción $\left(^{\circ}\right)$ & $46^{\circ}$ & $39^{\circ}$ & $34^{\circ}$ \\
\hline Cohesión $(\mathrm{kPa})$ & 64 & 38 & 10 \\
\hline
\end{tabular}

\section{Consecuencias en el diseño de las obras subterráneas}

El amplio rango de los resultados de los ensayos de resistencia al corte in situ tiene algunas consecuencias en el diseño de las obras subterráneas. Por ejemplo, al comparar los diseños obtenidos con la resistencia al corte más alto y los obtenidos con la resistencia al corte más bajo, ocurre lo siguiente:

- Para los muros-pantalla a construirse en las estaciones del Metro, se requeriría menor espesor y refuerzo, y menos profundidad de empotramiento para lograr mantener una estabilidad segura mientras más resistente sea el suelo. Esto podría conducir, incluso, a contemplar la posibilidad de construir las estaciones con muro-pilote, lo que podría resultar más económico que lo que se está considerando actualmente con la L2 del Metro. La estructura de concreto armado, para la resistencia al corte in situ más baja obtenida, podría resultar, por lo menos, $50 \%$ a $100 \%$ más cara que para el caso de la resistencia al corte in situ más alta.

- Para los túneles construidos como mina (con el mal llamado "método NATM"), sería posible construir las estructuras, en una fase, con diámetros más grandes mientras más resistente sea el suelo. De hecho, en el kilómetro 5 de la L2, construido como mina en la zona de Vitarte, se ha podido erigir el túnel en una fase con un diámetro de $\mathrm{D}=10 \mathrm{~m}$, lo cual alentaría la posibilidad, en caso sea necesario, de construir una estación tipo caverna (del orden de $\mathrm{D}=20 \mathrm{~m}$ ). De hecho, esto nos permite aceptar ya la posibilidad de que la construcción de los túneles (entre estaciones) se puede realizar en forma de mina, considerando también la opción con tuneladora, como estaba inicialmente planificado. Sin embargo, si nos remitimos al caso de la resistencia al corte in situ más baja obtenida, apenas se podría construir en una fase con un diámetro de $\mathrm{D}=3 \mathrm{~m}$, lo que significa que sería mucho más caro construir una estación en caverna. Por el contrario, para el caso de la resistencia al corte in situ más alta obtenida, se podría construir en una fase con un diámetro de hasta $\mathrm{D}=28 \mathrm{~m}$, lo que supondría que es relativamente simple construir una estación en caverna. 


\section{Marco teórico del comportamiento de una grava}

De acuerdo con varios investigadores, está ampliamente demostrado que las gravas no tienen cohesión para la "resistencia al corte residual" [7]. Sin embargo, los altos y empinados acantilados de la costa de Lima demuestran que hay una importante cohesión, aunque, probablemente, cohesión para la "resistencia al corte pico" (ver fig. 4). En estos acantilados, al lado de macizos firmes, compactos y empinados, hay grava coluvial formando taludes en el margen de $40^{\circ}$ a $45^{\circ}$ (ver foto 1 ), lo que demostraría que es el resultado de la falla de los macizos empinados que han alcanzado la resistencia al corte residual (falla en la superficie y por partes o gránulos), debido, probablemente, a acciones externas como viento, sismos, acción del hombre, etc.

La resistencia al corte pico es tanto mayor respecto a la resistencia al corte residual cuanto más densa es la grava, ocurriendo a deformaciones cada vez menores [7]. Esto quiere decir que las gravas muy densas tienen un comportamiento frágil (ver fig. 4) con una resistencia pico alta, lo que significa que la falla ocurre a una deformación baja que prácticamente podría no advertir de su ocurrencia (falla brusca). Por ello, es importante determinar la resistencia al corte pico y la cohesión, en particular para las gravas densas, para aplicarles un adecuado factor de seguridad que asegure la estabilidad. Esto es importante sobre todo cuando hay descarga en los suelos, como ocurre en las excavaciones de los túneles y estaciones de metro.

La curva envolvente de la resistencia al corte pico, en función de las presiones de confinamiento, suele ser cóncava hacia abajo (ver fig. 4), con una importante contribución de la dilatancia a presiones de confinamiento baja; y con una contribución de la trituración de las partículas a presiones de confinamiento alta [7] (ver fig. 4).

\section{Programa de Investigación Geotécnica Especial para las Gravas}

El objetivo de este programa es la obtención de las correlaciones de las propiedades mecánicas (de muy alto costo) con propiedades índice (de bajo costo), de tal manera que en el Programa de Investigación Geotécnica Masiva, se preste más atención a la obtención de abundante información de las propiedades índice (con una perspectiva estadística), minimizando, por otro lado, la ejecución de ensayos especiales para la obtención de las propiedades mecánicas. Este programa tendría que ser flexible en su definición, ya que las necesidades de diferentes tipos de ensayos pueden ir variando según los resultados parciales que se vayan obteniendo. El programa mínimo e inicial podría seguir los siguientes pasos:

1) Ubicación de unas cuantas decenas de calicatas profundas (del orden de $30 \mathrm{~m}$ ), aproximadamente, 20 a 40 calicatas, preferentemente ubicadas en parques (para no interrumpir el tráfico), en la zona del cono aluvial central del valle de Lima, que contiene abundante presencia de gravas.

2) Ejecución de ensayos MASW-1D para la determinación de las velocidades de onda de corte en el punto donde se excavaría la calicata, y antes de que el subsuelo sea alterado.

3) Excavación de cada calicata para la ejecución de ensayos a diferentes cotas o plataformas (pueden ser de 3 a 5 niveles diferentes). Si se encuentra nivel freático (como ocurriría en Callao), entonces, se interrumpiría la excavación de la calicata.

4) En cada plataforma, se procede a identificar los estratos representativos, a clasificar los suelos (grava GP, en su mayoría), a tomar densidades in situ con algún método ad hoc a la granulometría del suelo (considerar presencia de bolonería para la definición del método ad hoc), y a ejecutarse ensayos de corte directo in situ (ver fig. 3), en caja grande (se dispone, en medio, cajas de $70 \mathrm{~cm}$ ), con diferentes presiones de confinamiento similares a la profaundidad de interés, incluyendo presión nula para tratar de determinar la cohesión. Probablemente, varios de estos ensayos no vayan 
a ser válidos, debido a que la relación (tamaño-muestra / tamaño-máximo de partícula) podría ser menor que 5. Dependiendo de la frecuencia de estos casos, se podría analizar la posibilidad de agrandar las cajas de confinamiento de las muestras, o podrían repetirse los ensayos o, simplemente, registrar los ensayos para encontrar si hay alguna metodología de corrección. Finalmente, se cierra la calicata.

5) Debido a la dificultad en el control de las variables de los ensayos de corte directo in situ, se procede a ejecutar una serie de ensayos de corte directo en laboratorio, en caja grande $(60 \mathrm{~cm}$ disponible en la UNI-CISMID). Estos ensayos podrían ejecutarse para unas 4 a 8 curvas granulométricas representativas de todo el cono aluvial de Lima, de gravas GP, que se podrían caracterizar, por ejemplo, por el porcentaje de bolonería, el porcentaje de contenido de finos o el $\mathrm{D}_{50}$. Por tanto, la identificación de la caracterización más apropiada sería un tema a estudiar. Para cada una de estas curvas granulométricas, se podría compactar las muestras a unas 3 a 4 densidades diferentes. Entonces, se tendrían 12 a 32 muestras de diferentes granulometrías y densidades. Cada una de estas muestras se replicarían para ser sometidas a diferentes presiones de confinamiento (podría ser $0,2,4$ y $8 \mathrm{~kg} / \mathrm{cm}^{2}$ ). Con todos estos resultados, se podría buscar una correlación de la resistencia al corte (ángulo de fricción y cohesión) con la densidad y la variable que identifica al tipo de curva granulométrica, que son las variables más importantes para el caso de suelos granulares, a efectos de correlacionar con su resistencia al corte. No se considera la angulosidad de las partículas ni el origen geológico, pues todas provienen de una misma fuente (valle del Rímac). La anisotropía podría ser una variable importante, debido a que la esfericidad promedio es del orden de 0.74. Para el caso de Callao, convendría, además, realizar ensayos drenados, pero con las muestras saturadas.

6) Para el punto donde se ejecutó cada calicata profunda, se obtendría la resistencia al corte por correlación con las densidades in situ previamente obtenidas. Además, se usaría la data de la resistencia al corte in situ debidamente corregida por la presencia de bolonería dentro de la caja de cada ensayo. Luego, se buscaría correlacionar la resistencia al corte con las velocidades de onda de corte, aunque cabe advertir que estas correlaciones son demasiado dispersas para las gravas, según la literatura técnica [8]. Sin embargo, como se estaría investigando solo las gravas de Lima, entonces, quizás, estas correlaciones no lleguen a ser tan dispersas.

7) En cuanto a las propiedades deformacionales (módulo de elasticidad y de corte dinámico o a baja deformación), estas se podrían deducir a partir de las velocidades de onda de corte. Luego, se podría construir las curvas esfuerzo-deformación en base a curvas típicas para gravas densas, obtenidas de ensayos de corte cíclicos durante varias décadas en los principales centros de investigación del mundo.

8) En el futuro cercano, convendría que en la universidad (UNI-CISMID) se construya una cámara triaxial grande, de un diámetro $\mathrm{D}>1 \mathrm{~m}$, para obtener las propiedades mecánicas de las gravas con las variables mejor controladas y con la posibilidad de poder determinar la resistencia al corte pico y la residual, pues en los ensayos de corte directo es difícil precisarlos.

\section{Programa de Investigación Geotécnica Masiva para las Gravas}

A lo largo de cada línea de Metro a estudiarse, se podrían ejecutar los siguientes ensayos:

1) Geofísica MASW-2D (de ondas superficiales) para la determinación de la tomografía de la velocidad de onda de corte a lo largo de la línea, y hasta del orden de $30 \mathrm{~m}$ de profundidad. En el poco probable caso de que se requiera investigar a más profundidad, se podría usar la geofísica MAM (multitrepidaciones) que llega hasta $100 \mathrm{~m}$. Estos ensayos debieran ayudar, en principio, a 
sectorizar la línea de Metro, según el rango de propiedades mecánicas de las gravas. También, se podría usar, aunque con cierta reticencia para determinar por correlación, la resistencia al corte; y con regular precisión para la determinación de las propiedades deformacionales.

2) Se ejecutaría sondeos hasta de unos $10 \mathrm{~m}$ por debajo de la estructura subterránea proyectada, con el fin de detectar la presencia de otros suelos distintos a las gravas GP y el nivel freático (poco probable en la provincia de Lima). En los otros tipos de suelo más finos (SM, ML, CL, etc.) se ejecutarían los ensayos estándar para su clasificación y determinación de la resistencia al corte [4]. En los sondeos también se debiera ejecutar ensayos presiométricos a diferentes profundidades para la determinación de las propiedades deformacionales [4] y buscar su correlación con aquellas obtenidas con las velocidades de ondas de corte [9].

3) Se ejecutaría pocas calicatas profundas $(30 \mathrm{~m})$ debido a lo caras que son. Estas se ejecutarían en puntos donde no se interrumpa el tráfico. En estas pocas calicatas, se procedería a determinar, en diferentes niveles, la granulometría representativa, la densidad in situ y la resistencia al corte in situ. Se procuraría ejecutar, por lo menos, una calicata profunda por sector geotécnico (ver paso 1).

4) Como se observa en los pasos 1 al 3, el objetivo de los ensayos MASW-2D y de los ensayos en las calicatas profundas es determinar, de manera conjunta, las propiedades mecánicas de las gravas para cada sector geotécnico de la línea de Metro en estudio. El objetivo de los sondeos es la definición del perfil estratigráfico con la ayuda de las calicatas profundas, así como la determinación de la propiedades mecánicas de los otros tipos de suelo (¿SM, CL, etc.?) de muy escasa presencia.

\section{Referencias}

[1] Estudio de Preinversión a Nivel de Factibilidad del Proyecto: "Construcción de la Línea 2 y Ramal Av. Faucett-Gambeta de la Red Básica del Metro de Lima y Callao"-Ingeniería Básica del Proyecto, Proinversión, julio de 2013.

[2] U.S. National Committee on Tunnelling Technology, Geotechnical Site Investigation for Undergroung Projects, Washington DC, 1984.

[3] Proyectos Integrales para la Concesión de la Línea 3 de la Red Básica del Metro de Lima y Callao-Estudios Básicos de Ingeniería. Proinversión, mayo de 2015.

[4] Roy E. Hunt. Geotechnical Engineering Investigation Handbook. Second Edition. CRC Press Taylor \& Francis Group, 2005.

[5] Vallerga, Seed, Monismith, and Cooper, R. S., 1957, "Effect of Shape Size and Surface Roughness of Aggregate Particles on the Strength of Granular Materials", Special Technical Publication No. 212, ASTM.

[6] J. Coll Calderón, Futura Red del Metro y Trenes de Cercanías, CD-Lima-CIP, Dic.2015

[7] M. Maksimovic, Nonlinear failure envelope for coarse-grained soils Belgrade University, Yugoslavia.

[8] K.M. Rollins, M. D. Evans, N. B. Diehl and W. D. Daily, Shear Modulus and Damping Relationship for Gravels. Members ASCE, JGGE, May 1998

[9] J. Coll Calderón, Investigación Geotécnica para la Red del Metro de Lima. Congreso de Ingeniería Civil, Huaraz, diciembre de 2015. 


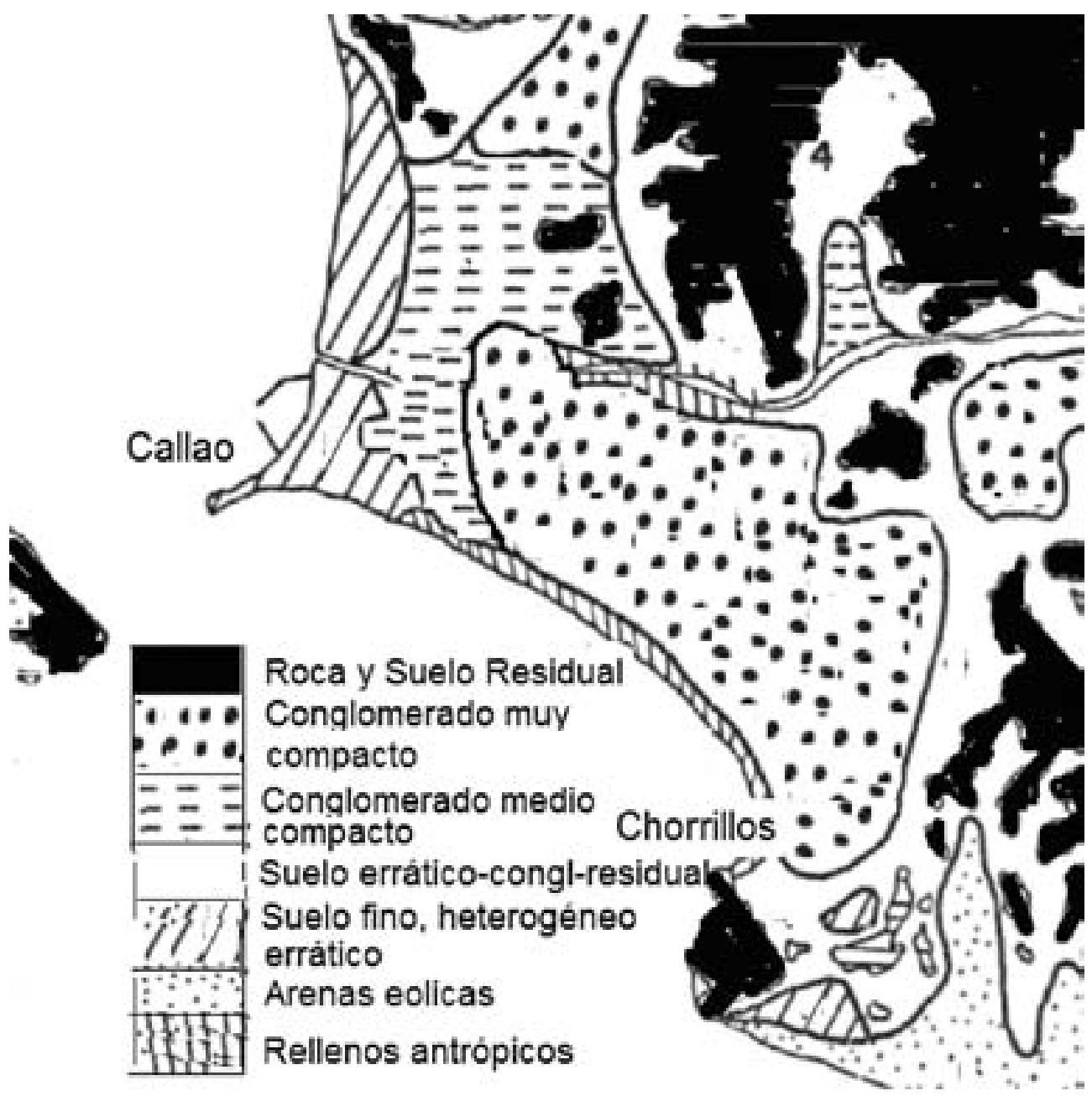

Fig. 1. Geología en planta en Lima Metropolitana.

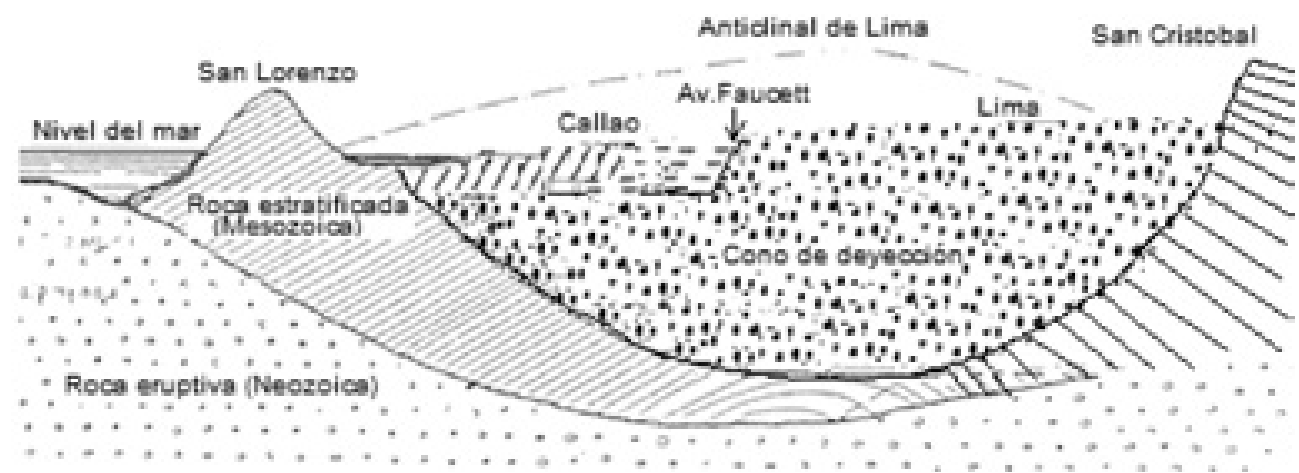

Fig. 2. Perfil geológico, de oeste a este, del centro de Lima Metropolitana. 


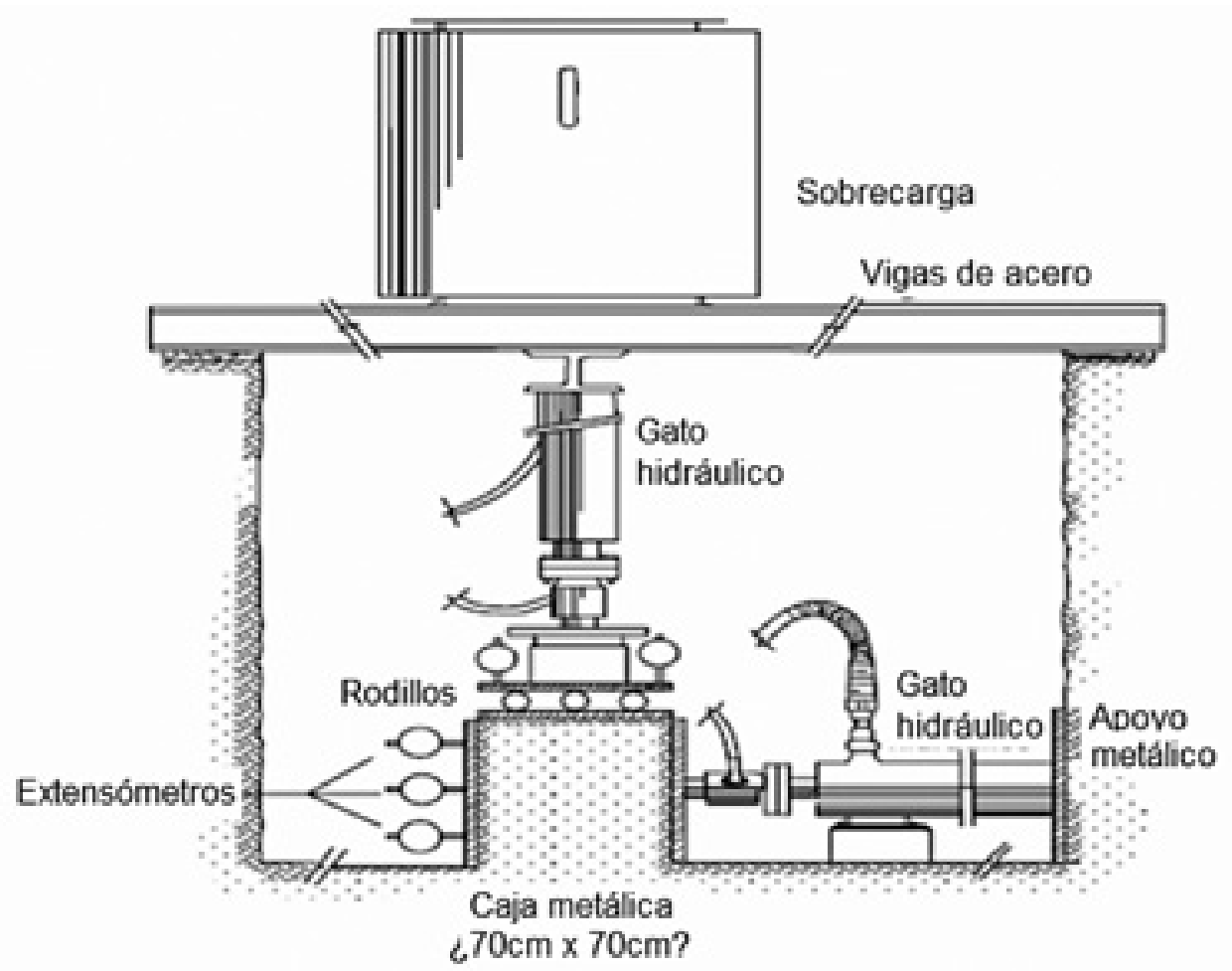

Fig. 3. Esquema del ensayo de corte directo in situ.
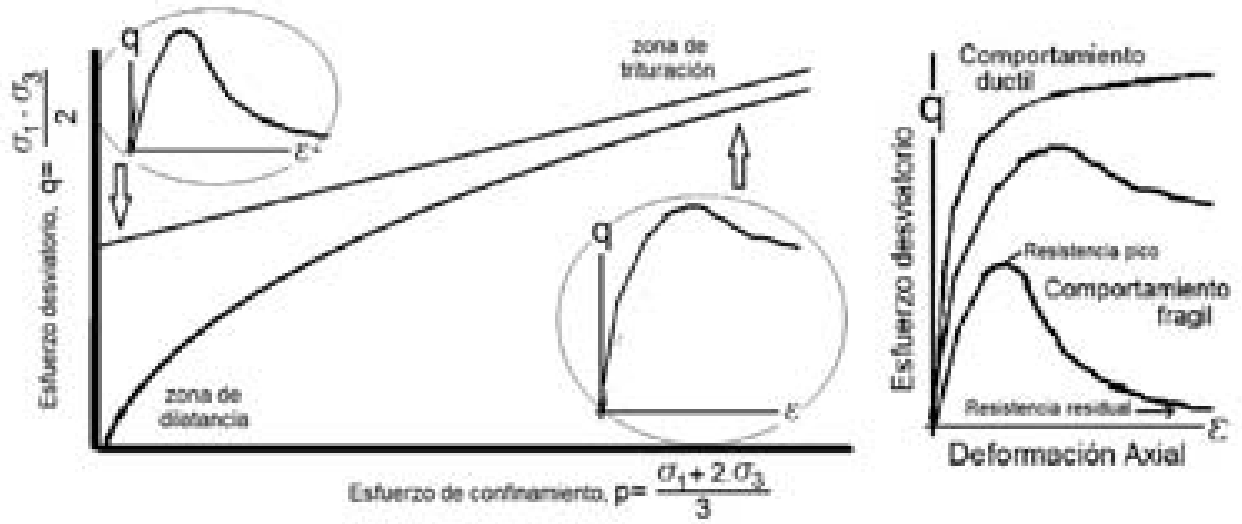

Fig. 4. Características del comportamiento mecánico de gravas compactas. 


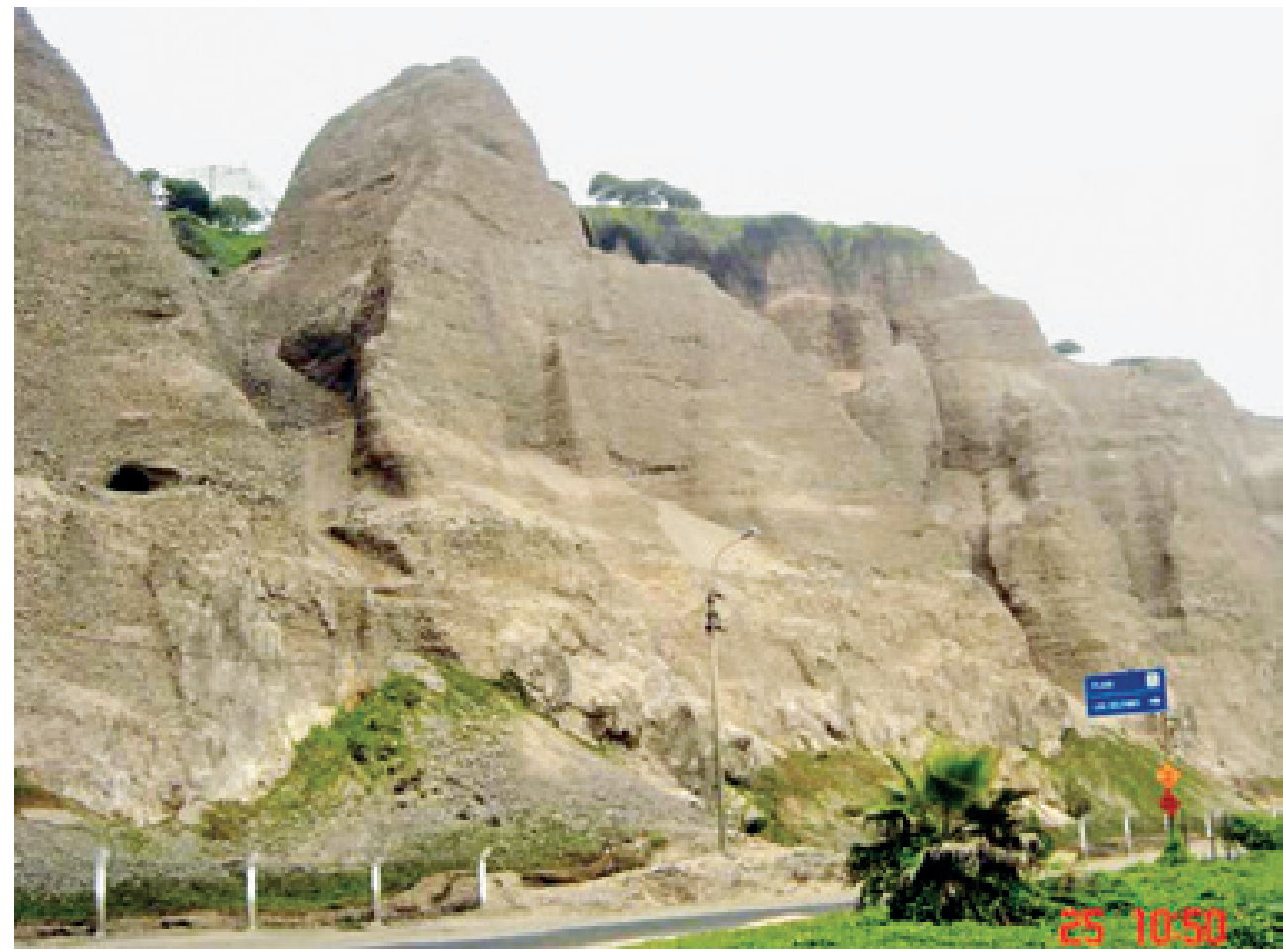

Foto 1. Vista del acantilado costero de Lima en la zona del conglomerado muy compacto. 
